\title{
RELAÇÃO ENTRE O ZINCO “DISPONÍVEL”, POR DIFERENTES EXTRATORES, E AS FRAÇÕES DE ZINCO EM AMOSTRAS DE SOLOS ${ }^{(1)}$
}

\author{
M. F. G. OLIVEIRA(2), R. F. NOVAIS ${ }^{(3)}, J$. C. L. NEVES ${ }^{(3)}$, \\ C. A. VASCONCELLOS ${ }^{(4)} \&$ V. M. C. ALVES ${ }^{(4)}$
}

\begin{abstract}
RESUMO
Para determinar o zinco "disponível" do solo para plantas, vários procedimentos de extração têm sido desenvolvidos. Uma alternativa utilizada no estudo de extratores do $\mathrm{Zn}$ "disponível" refere-se ao fracionamento do $\mathrm{Zn}$ total do solo, com vistas em entender suas reações no solo e o comportamento dos extratores. Este trabalho objetivou avaliar a dependência existente entre o teor de Zn disponível, por diferentes extratores, e as frações deste elemento no solo e características dos solos. Para isto, amostras de doze solos da camada de 0-20 cm de profundidade, correspondendo aos grandes grupos de Latossolo Vermelho-E scuro (LE), Latossolo Vermelho-Amarelo (LV), Latossolo Amarelo (LA), Podzólico Vermelho-Amarelo (PV) e Areia Quartzosa (AQ), receberam as doses de 0 e $20 \mathrm{mg} \mathrm{dm}^{-3}$ de $\mathrm{Zn}$ e foram incubadas por 30 dias. $O \mathrm{Zn}$ extraível por DTPA-TEA-CaCL 2 HCl (0,1 mol L-1), Mehlich-1 (M-1) e Mehlich-3 (M-3) foi determinado. Essas amostras dos solos foram também submetidas ao fracionamento de $\mathrm{Zn}$, determinando-se Zn trocável (Zntr), ligado à matéria orgânica (Znmo), ligado a óxido de manganês ( $\mathrm{ZnMn}$ ), ligado a óxido de ferro amorfo ( $\mathrm{ZnF}$ ea) e ligado a óxido de ferro cristalino ( $\mathrm{ZnFec}$ ). Concluiu-se que os extratores DTPA e M-3 revelaram maior sensibilidade às características do solo relacionadas com o fator capacidade (poder tampão). Os extratores $\mathrm{M}-1$ e $\mathrm{HCl}$ apresentaram menor sensibilidade e menor correlação com estas características, considerando seu maior poder de extração e conseqüente menor desgaste. A relação $\mathrm{Zn}$ recuperado pelo extrator $/ \mathrm{Zn}$ aplicado ao solo demonstrou ser a característica que melhor se correlacionou com características do solo relacionadas com o fator capacidade de $\mathrm{Zn}$. A fração de Zn trocável foi a maior responsável pela quantidade de $\mathrm{Zn}$ obtido pelos extratores testados. As frações de Zntr, Znmo, ZnMn, ZnFea e ZnFec não foram suficientes para explicar, em todos os casos, o zinco recuperado pelos extratores.
\end{abstract}

Termos de indexação: fracionamento de zinco, micronutriente, análise de solo, fator capacidade de zinco.

(1) Recebido para publicação em setembro de 1998 e aprovado em junho de 1999.

(2) Engenheiro-Agrônomo, Doutorando em Solos e Nutrição de Plantas, Departamento de Ciência do Solo, Universidade Federal de Lavras (UFLA). CEP 37200-000 Lavras (MG).

(3) Professor do Departamento de Solos, Universidade Federal de Viçosa. CEP 36571-000 Viçosa (MG).

(4) Pesquisador da Embrapa Milho e Sorgo. Rodovia MG 424 Km 65, Caixa Postal 151, CEP 35701-970 Sete Lagoas (MG). 


\title{
SUMMARY: RELATIONSHIP BETWEEN THE ZINC AVAILABLE BY DIFFERENT EXTRACTING SOLUTIONS AND ZINC FRACTIONS IN SOIL SAMPLES
}

\begin{abstract}
Many extracting procedures for theplant-availablezinc havebeen devel oped. Total soil Zn fractionation has been used as an attempt to understand the reactions of this nutrient in soils and the behaviour of the extracting procedures. This work evaluated the influence of soil characteristics on the concentration of $Z n$ extracted by different procedures and on soil $\mathrm{Zn}$ fractions. Samples were collected from the $0-20 \mathrm{~cm}$ surface layer of twelve soils, representing thegreat soil groups of Dark-Red Latosol (LE), Red-Yellow Latosol (LV), Yellow Latosol (LA), Red-Yellow Podzolic (PV), and Quartzi Psaments (AQ). Thesoil samples received 0 and $20 \mathrm{mg} \mathrm{dm}^{-3}$ of $\mathrm{Zn}$ and were incubated for 30 days. Soil $\mathrm{Zn}$ concentration was determined after extraction with DTPA-TEA-CaCL $2,0.1$ mol L-1 $\mathrm{HCl}$, Mehlich-1 (M-1) and Mehlich-3 (M-3). Zinc fractionation was also carried out to determine exchangeable Zn (ExZn), Zn tied up to organic matter (OmZn), to manganese oxide (MnZn), to amorphous iron oxide(AFeZn), and to crystall lineiron oxide(CFeZn). DTPA and M-3 weretheextractants with larger sensitivity to the soil characteristics related to the capacity factor (buffering power). The $\mathrm{M}-1$ and $\mathrm{HCl}$ extractants presented lower sensitivity and lower corre ation with these characteristics due to their larger extraction power. The ratio $\mathrm{Zn}$ recovered by the extractant/ Zn applied to thesoil showed thebest correlation with soil characteristics related to the $Z n$ capacity factor. Exchangeable $Z n$ fraction was thelargest sourceof this nutrient to thetested extractants. Thefractions of ExZn, OmZn, MnZn AF eZn and CFeZn did not account for thezinc recovered by the extractants, in all cases.
\end{abstract}

Index terms: zinc fractionation, mi cronutrient, soil analyses, zinc capacity factor.

\section{INTRODUÇÃO}

A pesquisa sobre a disponibilidade de nutrientes, particularmente de micronutrientes, em solos tem apresentado resultados contraditórios. I sto se deve, em grande parte, às numerosas inter-relações entre características dos sol os ea porção do nutriente tida como "disponível". Tais resultados indicam a necessidade de mai or refinamento de interpretações que melhor expressem a disponibilidade de micronutrientes e a resposta de plantas.

De modo geral, os extratores utilizados para Zn, e para os outros micronutrientes, podem ser agrupados de acordo com suas propriedades químicas em: sais neutros, ácidos, bases e agentes quelatantes ou complexantes (Wu et al., 1991). Os sais neutros contêm cátions com característica química similar ao elemento que se deseja extrair, promovendo o seu deslocamento para a solução extratora. Os extratores ácidos, como o Mehlich-1 e o $\mathrm{HCl}$ 0,1 mol L-1, atuam por dissolução (Wu et al., 1991). Entre os métodos de extração com agente complexante, citam-se DTPA-TEA-CaCl 2 e o Mehlich-3 (Mehlich, 1984).

A adsor ção, com grande energia, do Zn nos solos, particularmente naqueles com argila predominantemente oxídica (Kal basi et al., 1978; Cavallaro \& McBride, 1984) ou em condições de pH mais elevado (Lindsay, 1972; Bar-Yosef, 1979), tem sido considerada importante causa da sua restrita disponibilidade para as plantas. Assim, a restrita dessorção do Zn do solo nessas condições fez com que seus níveis críticos (NCs) pelos extratores Mehlich-1 e HCl 0,1 mol L-1, para o cultivo de milho em amostras de solos de cerrado, mostrassem-se inversamente relacionados com características dos solos que refletem o seu poder tampão ou fator capacidade de Zn (FCZn) (Couto et al., 1992). É provável que o desgaste do extrator, como largamente observado para P (Novais \& Smyth, 1999), seja responsável por essa dependência dos valores dos NCs ao FCZn.

O entendimento da disponibilidade de micronutrientes em solos tem melhorado pelo uso de técnicas de extrações seqüenciais, permitindo observar a distribuição do elemento em diferentes frações no sol o (Sims, 1986; Soon, 1994; A humada et al., 1997; Chowdhury et al., 1997). Em geral, esquemas de fracionamento aval iam a concentração do elemento em solução, trocável, organicamente complexado e ligado a óxidos. Amostras de 20 solos de Nova Zelândia foram submetidas ao fracionamento de Zn, mostrando que a participação de cada fração, em relação ao Zn total, variou entre solos. $\mathrm{Na}$ média, 3\% de $\mathrm{Zn}$ total encontrou-se na forma trocável , 5\% ligado à matéria orgânica (MO), 9, 18 e $24 \%$ associados a óxidos de $\mathrm{Mn}$, de Fe amorfo e 
cristal ino, respectivamente, e $40 \%$ na fração resi dual. Extratores do Zn disponível correlacionaram-se mais intimamente com o Zn trocável e com oligado a MO (Chowdhury et al., 1997).

Cada vez mais, tem-se tentado um refinamento da técnica de fracionamento para o desenvol vimento de um método adequado ao maior número possível de classes de solos.

Este trabalho objetivou avaliar as relações existentes entre os teores de Zn "disponível" pelos extratores DTPA-TEA-CaCl 2 , Mehlich-1, Mehlich3 e HCl e as diferentes frações desse el emento no solo e seus atributos.

\section{MATE RIAL E MÉTODOS}

Amostras de 12 solos da camada de $0-20 \mathrm{~cm}$ de profundidade foram coletadas nos municípios de Campina Verde, Sete Lagoas, Paracatu, Patrocínio, Três Marias, U beraba e Ponte Nova, no estado de Minas Gerais, e nos municípios de Aracruz e de Açailândia, nos estados do Espírito Santo e do Maranhão, respectivamente. Esses solos compreendem uma ampla variação textural, correspondendo aos grandes grupos de L atossolo VermelhoEscuro (LE), Latossolo Vermel ho-Amarelo (LV), Latossolo Amarelo (LA), Podzólico VermelhoAmarelo (PV) e Areia Quartzosa (AQ). Essas amostras foram secas ao ar, passadas em peneira de $2 \mathrm{~mm}$, homogeneizadas (TFSA) e submetidas a anál ises químicas, físicas e mineralógicas (Quadros 1 e 2).

\section{Teor de zinco "disponível”}

Foram retiradas seis subamostras de $100 \mathrm{~cm}^{3}$ de cada amostra de solo. Três subamostras receberam uma dose de $20 \mathrm{mg} \mathrm{dm}^{-3}$ de $\mathrm{Zn}$ na forma de $\mathrm{ZnCl}_{2}$, reagente p.a., e as outras três não, sendo todas elas incubadas por um período de 30 dias à temperatura ambiente. As subamostras foram, então, secas, passadas em peneira de $2 \mathrm{~mm}$ e submetidas à análise por quatro extratores:

Mehlich-1 ( $\mathrm{HCl} \quad 0,05 \mathrm{~mol} \mathrm{~L}^{-1}$ e $\mathrm{H}_{2} \mathrm{SO}_{4}$ $0,0125 \mathrm{~mol} \mathrm{~L}^{-1}$ ): $10 \mathrm{~cm}^{3}$ TFSA $+100 \mathrm{~mL}$ de solução extratora foram agitados por $5 \mathrm{~min}$, deixados em repouso por $16 \mathrm{~h}$ e retirada uma alíquota para determinação de Zn (Defelipo \& Ribeiro, 1981).

$\mathrm{HCl}\left(0,1 \mathrm{~mol} \mathrm{~L}^{-1}\right): 2 \mathrm{~cm}^{3}$ TFSA $+20 \mathrm{~mL}$ de solução extratora foram agitados por $5 \mathrm{~min}$, centrifugados, retirado o sobrenadante, repetindo-se o processo por duas vezes. Os sobrenadantes foram filtrados e reunidos em um único extrato, homogeneizado e, então, retirada uma alíquota para determinação de Zn (Wu et al., 1991).

DTPA-TEA-CaCl 2 (ácido dietileno-triaminopentacético 0,005 mol L-1, trietanolamina $0,1 \mathrm{~mol} \mathrm{~L}^{-1} \mathrm{e}$ $\mathrm{CaCl}_{2} \cdot 2 \mathrm{H}_{2} \mathrm{O}$ 0,01 mol L-1, pH ajustado para 7,3):
$10 \mathrm{~cm}^{3}$ deTFSA $+20 \mathrm{~mL}$ de solução extratora foram agitados por $2 \mathrm{~h}$, filtrados e, então, retirada uma alíquota para determinação de Zn (Lindsay \& Norvell, 1978).

Mehlich-3 $\left(\mathrm{CH}_{3} \mathrm{COOH} 0,2 \mathrm{~mol} \mathrm{~L}-1, \mathrm{NH}_{4} \mathrm{NO}_{3}\right.$ $0,25 \mathrm{~mol} \mathrm{~L}^{-1}, \mathrm{NH}_{4} \mathrm{~F} 0,015 \mathrm{~mol} \mathrm{~L}^{-1}, \mathrm{HNO}_{3} 0,013 \mathrm{~mol} \mathrm{~L}^{-1} \mathrm{e}$ ácido etileno-diaminopentacético 0,001 mol L-1): $3 \mathrm{~cm}^{3}$ de TFSA $+30 \mathrm{~mL}$ de solução extratora foram agitados por 5 min, filtrados e retirada uma alíquota para determinação de Zn (Mehlich, 1984).

O Zn obti do pel os diferentes métodos de extração foi dosado por espectrofotometria de absorção atômica.

\section{Método de fracionamento de zinco do solo}

O processo de fracionamento consistiu na separação do $Z n$ presente no solo nas formas $Z n$ trocável (Zntr), Zn ligado à matéria orgânica (Znmo), Zn ligado a óxido de manganês (ZnMn), Zn ligado a óxido de ferro amorfo (ZnF ea) e Zn ligado a óxido de ferro cristalino (ZnFec), segundo Sims (1986). Esse procedimento foi usado nas subamostras com e sem aplicação de $20 \mathrm{mg} \mathrm{dm}^{-3}$ deZn, utilizadas na extração do "disponível". Estimou-se, também, oZn retido no solo sob outras formas que não as citadas zzinco residual, obtido pela diferença: 20 - [Zn total (somatório de todas as frações anteriores) na dose de $20 \mathrm{mg} \mathrm{dm}^{-3}$ ] + Zn total na dose 0 de $\mathrm{Zn}$ \}. Todas as frações foram obtidas da mesma amostra em um processo de extração seqüencial.

Zinco trocável: $5 \mathrm{~cm}^{3}$ TFSA foram agitados por $2 \mathrm{~h}$ com $20 \mathrm{~mL}$ de uma solução $\mathrm{Mg}\left(\mathrm{NO}_{3}\right)_{2} 1 \mathrm{~mol} \mathrm{L-1}$ em um tubo de centrífuga de $100 \mathrm{~mL}$. A amostra foi centrifugada por $10 \mathrm{~min}$. O sobrenadante foi separado, e adicionaram-se $20 \mathrm{~mL}$ de água deionizada ao solo, agitando-se por mais 3 min e centrifugado-se. Os dois sobrenadantes foram homogeneizados, determinando-se Zn.

Zinco ligado à matéria orgânica: $10 \mathrm{~mL}$ de solução de $\mathrm{NaClO} 53 \mathrm{~g} \mathrm{~L}-1$, pH 8,5, foram adicionados ao solo residual, no tubo de centrífuga. A amostra foi aquecida em banho-maria até $100^{\circ} \mathrm{C}$ por $30 \mathrm{~min}$, centrifugada e o sobrenadante filtrado em papel de filtro Whatman 42. Tal procedimento foi repetido uma vez mais e os filtrados combinados. Depois, foram adicionados $10 \mathrm{~mL}$ de água deionizada à amostra no tubo de centrífuga, agitando-se por 3 min, centrifugando-se, filtrando-se e misturandose o filtrado com o extrato $\mathrm{NaClO}$. O sol o no tubo de centrífuga, do procedimento anterior, foi seco ao ar, triturado e passado em uma peneira de $0,5 \mathrm{~mm}$. $\mathrm{O}$ material obtido foi utilizado na extração da fração de Zn ligado a óxido de manganês.

Zinco ligado a óxido de manganês: $2,5 \mathrm{~g}$ de solo (resíduo) do procedimento anterior e $25 \mathrm{~mL}$ de uma solução $\mathrm{NH}_{2} \mathrm{OH} . \mathrm{HCl} 0,1 \mathrm{~mol} \mathrm{~L}-1, \mathrm{pH} \mathrm{2,0}$, foram agitados por $30 \mathrm{~min}$ em tubo de centrífuga. A amostra foi, então, centrifugada e filtrada como 
descrito anteriormente e o extrato reservado para determinação de Zn. Vinte ecinco mililitros de água dei onizada foram adicionados, agitados com o solo por 3 min e descartados.

Zinco ligado a óxido de ferro amorfo: $25 \mathrm{~mL}$ de uma solução $\mathrm{N} \mathrm{H}_{2} \mathrm{OH}$. $\mathrm{HCl} 0,25 \mathrm{~mol} \mathrm{~L}-1+\mathrm{HCl}$ $0,25 \mathrm{~mol} \mathrm{~L}^{-1}$ foram adicionados ao solo no tubo de centrífuga eaquecidos por $30 \mathrm{~min}$ a $50^{\circ} \mathrm{C}$, em banhomaria. As amostras foram centrifugadas, filtradas e lavadas como no processo anterior e o extrato reservado para determinação de Zn.

Zinco ligado a óxido de ferro cristalino: $25 \mathrm{~mL}$ de uma solução $\left(\mathrm{NH}_{4}\right)_{2} \mathrm{C}_{2} \mathrm{O}_{4} 0,2 \mathrm{~mol} \mathrm{~L}-1+\mathrm{H}_{2} \mathrm{C}_{2} \mathrm{O}_{4}$ $0,2 \mathrm{~mol} \mathrm{L-1}, \mathrm{pH} 3,0$, foram adicionados ao sol o notubo de centrífuga e aquecidos por $30 \mathrm{~min}$ a $100^{\circ} \mathrm{C}$, em banho-maria, e agitados ocasionalmente. As amostras foram, então, centrifugadas, filtradas como já descrito, determinando-se o Zn.
A determinação de $Z n$ foi feita por espectrofotometria de absor ção atômica nos extratos de cada uma das frações de Zn.

\section{Procedimento estatístico}

O experimento constou de um fatorial de $12 \times 2$, correspondendo a solos e doses de $\mathrm{Zn}$, respectivamente, em um del ineamento inteiramentecasual izado, com três repetições.

Os teores de $\mathrm{Zn}$ obtidos pelos extratores e pelo fracionamento foram submetidos a análises de correl ação simples eanál ises de variância, utilizandose o SAEG (Sistema de Análises Estatísticas e Genéticas) e regressões múltiplas realizadas no sistema SAS. Correlacionaram-se os teores de $\mathrm{Zn}$ obtidos pel os extratores DTPA, $\mathrm{HCl}$, Mehlich-1 (M-1) e Mehlich-3 (M-3) com as características dos solos relacionadas com sua capacidade tampão.

\section{Quadro 1. Características químicas e físicas das amostras dos solos de Campina Verde (CV-AQd e CV- LEm), Três Marias (TM-LEm e TM-LVm), Aracruz (ARA-LA), Açailândia (AÇA-LA), (UBE 1-LVa e UBE2- LVa), Paracatu (PAR-LV), Sete Lagoas (SL-LE), Patrocínio (PAT-LV)* e Ponte Nova (PN-PV)}

\begin{tabular}{|c|c|c|c|c|c|c|c|c|c|c|c|c|}
\hline \multirow{2}{*}{ Característica } & \multicolumn{12}{|c|}{ Solo ${ }^{(a)}$} \\
\hline & CV-AQd & CV-LE m & TM-LEm & TM-LVm & ARA-LA & AÇA-LA & UBE 1-L Va & UBE 2-LVa & PAR-LV & SL-LE & PAT-LV & PN-PV \\
\hline $\mathrm{pH}$ em $\mathrm{H}_{2} \mathrm{O} 1: 2,5$ & 5,05 & 4,97 & 4,64 & 4,99 & 4,40 & 5,03 & 5,34 & 5,00 & 4,70 & 4,38 & 4,64 & 4,87 \\
\hline $\mathrm{pH}$ em KCl $1 \mathrm{~mol} \mathrm{~L}^{-1} 1: 2,5$ & 3,84 & 4,83 & 4,92 & 4,15 & 3,69 & 4,00 & 4,04 & 4,02 & 4,07 & 3,83 & 4,21 & 4,92 \\
\hline P Total, $\mathrm{mg} \mathrm{dm}^{-3}(1)$ & 64,1 & 243,0 & 116,1 & 41,0 & 64,4 & 94,4 & 298,0 & 363,1 & 228,1 & 934,0 & 439,6 & - \\
\hline P Mehlich-1, mg dm-3 (2) & 2,9 & 1,3 & 0,7 & 0,2 & 0,3 & 1,0 & 0,2 & 0,2 & 0,5 & 1,3 & 0,2 & 1,27 \\
\hline P Mehlich-3, mg dm-3 (3) & 3,7 & 1,8 & 1,3 & 0,3 & 0,3 & 1,5 & 0,8 & 1,0 & 0,6 & 2,7 & 0,0 & - \\
\hline P Olsen, $\mathrm{mg} \mathrm{dm}^{-3}$ (5) & 1,2 & 1,5 & 1,5 & 1,2 & 0,8 & 0,8 & 3,1 & 3,1 & 1,4 & 0,5 & 0,8 & - \\
\hline $\mathrm{P}$ remanescente, $\mathrm{mg} \mathrm{L}^{-1}$ (6) & 37,1 & 21,1 & 38,1 & 29,9 & 29,9 & 46,1 & 3,4 & 4,8 & 4,6 & 3,2 & 1,8 & 31,6 \\
\hline $\mathrm{CMAP}, \mathrm{mg} \mathrm{cm}^{-3}(7)$ & 0,390 & 0,659 & 0,335 & 0,434 & 0,453 & 0,299 & 1,673 & 1,887 & 1,728 & 1,757 & 1,936 & ; - \\
\hline CMAS, $\mathrm{mg} \mathrm{cm}^{-3}$ (7) & 0,011 & 0,063 & 0,012 & 0,067 & 0,064 & - & 0,188 & 0,188 & 0,215 & 0,199 & 0,279 & - \\
\hline $\mathrm{SiO}_{2}, \mathrm{~g} \mathrm{~kg}^{-1 / 1}$ & 46,2 & 77,2 & 45,8 & 38,4 & 138,6 & 112,8 & 164,8 & 88,2 & 235,8 & 247,6 & 67,0 & - \\
\hline $\mathrm{Al}_{2} \mathrm{O}_{3}, \mathrm{~g} \mathrm{~kg}^{-1}(8)$ & 35,7 & 66,3 & 51,0 & 81,6 & 128,6 & 86,7 & 327,5 & 363,2 & 360,7 & 277,8 & 257,0 & - \\
\hline $\mathrm{Fe}_{2} \mathrm{O}_{3}, \mathrm{~g} \mathrm{~kg}^{-1(8)}$ & 26,9 & 68,9 & 23,0 & 25,0 & 29,9 & 24,0 & 87,9 & 58,8 & 81,8 & 116,8 & 141,2 & - \\
\hline $\mathrm{TiO}_{2}, \mathrm{~g} \mathrm{~kg}^{-1}(8)$ & 5,4 & 23,0 & 8,8 & 9,9 & 19,3 & 8,2 & 18,8 & 22,5 & 12,3 & 10,3 & 7,8 & - \\
\hline $\mathrm{Ca}^{2+}, \mathrm{mmol}_{\mathrm{c}} \mathrm{dm}^{-3}(9)$ & 1,8 & 2,4 & 0,0 & 0,0 & 1,9 & 10,9 & 0,8 & 0,0 & 1,0 & 9,4 & 1,9 & 5,3 \\
\hline $\mathrm{Mg}^{2+}, \mathrm{mmol}_{\mathrm{c}} \mathrm{dm}^{-3}(9)$ & 1,7 & 4,2 & 0,4 & 0,3 & 1,0 & 2,3 & 0,9 & 0,6 & 1,3 & 1,5 & 1,6 & 1,3 \\
\hline $\mathrm{H}+\mathrm{Al}, \mathrm{mmol}_{\mathrm{c}} \mathrm{dm}^{-3}(10)$ & 46,4 & 52,2 & 31,7 & 24,8 & 36,4 & 29,4 & 78,9 & 82,8 & 78,9 & 128,4 & 86,6 & 39,0 \\
\hline $\mathrm{S}, \mathrm{mmol}_{\mathrm{c}} \mathrm{dm}^{-3}$ & 4,8 & 7,8 & 0,9 & 0,6 & 3,2 & 13,5 & 2,5 & 1,4 & 2,4 & 11,7 & 5,4 & 7,6 \\
\hline CTC efetiva, $\mathrm{mmol}_{\mathrm{c}} \mathrm{dm}^{-3}$ & 16,1 & 21,9 & 8,4 & 8,1 & 18,2 & 26,4 & 11,5 & 12,2 & 11,3 & 29,1 & 10,6 & 11,6 \\
\hline CTC total, $\mathrm{mmol}_{\mathrm{c}} \mathrm{dm}^{-3}$ & 62,5 & 74,1 & 40,1 & 32,9 & 54,6 & 55,8 & 90,4 & 95,0 & 90,2 & 157,5 & 97,2 & 46,6 \\
\hline $\mathrm{V} \%$ & 7,7 & 10,5 . & 2,2 & 1,8 & 5,9 & 24,2 & 2,8 & 1,5 & 2,7 & 7,4 & 5,6 & 16,8 \\
\hline $\mathrm{m} \%$ & 70,2 & 64,4 & 89,3 & 92,6 & 82,4 & 51,1 & 78,23 & 88,5 & 78,8 & 59,8 & 49,1 & 34,6 \\
\hline C.O. $\mathrm{g} \mathrm{kg}^{-1(11)}$ & 5,1 & 8,2 & 5,3 & 3,0 & 2,5 & 7,0 & 22,8 & 21,3 & 21,1 & 21,3 & 28,5 & 6,5 \\
\hline Areia grossa, $\mathrm{g} \mathrm{kg}^{-1}$ (12) & 580 & 520 & 500 & 510 & 460 & 500 & 110 & 150 & 30 & 80 & 180 & 500 \\
\hline Areia fina, $\mathrm{g} \mathrm{kg}^{-1}(12)$ & 290 & 230 & 360 & 300 & 170 & 190 & 60 & 60 & 80 & 70 & 40 & 220 \\
\hline Silte, $\mathrm{g} \mathrm{kg}^{-1}(12)$ & 10 & 20 & 20 & 30 & 20 & 100 & 160 & 190 & 210 & 130 & 240 & 60 \\
\hline Argila, $\mathrm{g} \mathrm{kg}^{-1}(12)$ & 120 & 230 & 120 & 160 & 350 & 210 & 670 & 600 & 680 & 720 & 540 & 220 \\
\hline Capac. Campo, kg kg ${ }^{-1(13)}$ & 0,15 & 0,19 & 0,15 & 0,18 & 0,18 & 0,19 & 0,33 & 0,35 & 0,35 & 0,37 & 0,37 & 0,18 \\
\hline Dens. A parente, $\mathrm{g} \mathrm{cm}^{-3}$ (6) & 1,30 & 1,25 & 1,39 & 1,38 & 1,31 & 1,31 & 0,98 & 0,96 & 0,94 & 0,96 & 1,00 & 1,22 \\
\hline
\end{tabular}

(a) Fonte: Com a exceção do PN - PV, amostras dos demais solos foram as mesmas utilizadas e analisadas por Fernández R. (1994). (1) Ataque $\mathrm{HNO}_{3} / \mathrm{HClO}_{4}$ (8:3) (v/v) (LANARV/SNAD, 1983). (2) Extrator: Mehlich-1 (Defelipo \& Ribeiro, 1981). (3) Extrator: Mehlich-3 (Mehlich, 1984). ${ }^{(4)}$ Extrator: Bray-1 (Bray \& Kurtz, 1945). ${ }^{(5)}$ Extrator: Olsen (Alvarez V., 1982). ${ }^{(6)}$ (Alvarez V., 1982). ${ }^{(7)}$ CMAP = Capacidade máxima de adsorção de P; CMAS = capacidade máxima de adsorção de S (Alvarez V., 1985). ${ }^{(8)}$ Ataque sulfúrico (Vettori, 1969). ${ }^{(9)}$ Extrator: KCl 1 mol L-1 (Defelipo \& Ribeiro, 1981). ${ }^{(10)}$ Extrator: CaOAc 0,5 mol L-1 , pH 7,0 (Defelipo \& Ribeiro, 1981). (11) Mé todo Walkley-Black (Defelipo \& Ribeiro, 1981). ${ }^{(12)}$ Método da pipeta (EMBRAPA, 1979). ${ }^{(13)}$ Método da coluna (Fernandes \& Sykes, 1968). 
Quadro 2. Composi ção mineralógica da fração argila, [caulinita (Ct), gi bsita (Gb) e goethita (Gt)], calculada a partir dos teores de óxidos obtidos pelo ataque sulfúrico e valores de $\mathrm{Ki}$, $\mathrm{Kr}$ e de relações de óxidos de amostras dos solos

\begin{tabular}{|c|c|c|c|c|c|c|c|}
\hline \multirow{2}{*}{ Solo(1) } & \multicolumn{3}{|c|}{ Composição mineralógica ${ }^{(2)}$} & \multirow{2}{*}{$\mathbf{K i}$} & \multirow{2}{*}{$\mathbf{K r}$} & \multicolumn{2}{|c|}{ Relações moleculares } \\
\hline & Ct & $\mathbf{G} \mathbf{b}$ & Gt & & & $\mathrm{Al}_{2} \mathrm{O}_{3} / \mathrm{Fe}_{2} \mathrm{O}_{3}$ & $\mathrm{SiO}_{3} / \mathrm{Fe}_{2} \mathrm{O}_{3}$ \\
\hline & . & $\mathrm{g} \mathrm{kg}^{-1}$ & - & & & & \\
\hline$C V-A Q d$ & 86 & - & 37 & 2,20 & 1,48 & 2,08 & 4,56 \\
\hline CV-LEm & 165 & - & 67 & 1,97 & 1,18 & 1,51 & 2,90 \\
\hline TM-LE m & 98 & 16 & 28 & 1,52 & 1,18 & 3,47 & 5,29 \\
\hline TM-LVm & 83 & 73 & 29 & 0,80 & 0,67 & 5,11 & 4,08 \\
\hline ARA-LA & 298 & 14 & 22 & 1,83 & 1,60 & 6,94 & 12,69 \\
\hline$A C ̧ A-L A$ & 243 & - & 30 & 2,21 & 1,88 & 5,65 & 12,48 \\
\hline U BE 1-LVa & 354 & 284 & 96 & 0,85 & 0,73 & 5,83 & 4,98 \\
\hline UBE 2-LVa & 190 & 438 & 55 & 0,41 & 0,37 & 9,66 & 3,98 \\
\hline PAR-LV & 507 & 243 & 97 & 1,11 & 0,97 & 6,90 & 7,66 \\
\hline SL-LE & 143 & 101 & 143 & 1,51 & 1,19 & 3,72 & 5,63 \\
\hline PAT-LV & 144 & 304 & 177 & 0,44 & 0,33 & 2,85 & 1,26 \\
\hline
\end{tabular}

(1) Fonte: Fernández R. (1994). O solo PN-PV não foi analisado. ${ }^{(2)}$ Segundo método de Resende et al. (1987).

As regressões múltiplas foram ajustadas por meio dos teores de $\mathrm{Zn}$ obtidos pel os extratores na dose de 0 e $20 \mathrm{mg} \mathrm{dm}^{-3}$, em função dos teores obtidos pelo fracionamento. Para calcular as regressões, foram utilizadas apenas as frações de Zn queapresentaram coeficiente de correlação entre si inferior a 0,8, eliminando-se, assim, os problemas de interpretação gerados por possível colinearidade entre frações de Zn. As equações de regressão múltipla tiveram a significância dos coeficientes testada por meio do quadrado médio do resíduo da análise de variância.

Utilizou-se, também, um procedimento para a determinação de "outliers", valores observados que apresentem 2,5 vezes ou mais a variância observada no experimento. Todo "outlier" detectado foi retirado do model o e a equação novamente calculada. Após a eliminação dos "outliers", procedeu-se à el iminação das variáveis independentes que não apresentavam coeficiente significativo dentro da equação. Optouse, também, pel o procedimento "sel ectium-stepwise" que fornece os valores de $\mathrm{R}^{2}$ parciais de cada um dos coeficientes significativos da equação. Finalmente, determinou-se a significância da falta de ajustamento dos model os de regressão.

\section{RESULTADOS E DISCUSSÃO}

O teor médio de $\mathrm{Zn}$ na dose de $20 \mathrm{mg} \mathrm{dm}^{-3}$ foi maior para os extratores $\mathrm{HCl}$ e $\mathrm{M}-1$, seguidos pelo M-3 e, por último, pelo DTPA, como indicado pelas relações $Z n$ recuperado/Zn adicionado, para o conjunto de solos (Quadro 3). Essa extração diferencial é resultante dos diferentes mecanismos de extração, em razão, principalmente, da composi ção química dos extratores (Wu et al., 1991).
A admissão da linearidade para o $Z n$ recuperado como variável do aplicado para os extratores utilizados é baseada em outros trabalhos, em que diversos autores, trabalhando com diferentes doses de $\mathrm{Zn}$ e os mesmos extratores utilizados neste trabalho, obtiveram uma recuperação (extração) também linear (Singh et al., 1994; Akram et al., 1995; Davis et al., 1995; Menezes, 1997).

Os extratores $\mathrm{HCl}$ e $\mathrm{M}-1$ apresentaram as menores variabilidades quanto à extração, considerando o conjunto desolos, como indicado pelos menores coeficientes de variação, 10,63 e 11,10\%, respectivamente (Quadro 3), indicando a menor sensi bilidade desses extratores à capacidade tampão dos solos, dado o provável menor desgaste desses dois extratores, como evidenciam N ovais \& Smyth (1999) para fósforo.

Correlações entre os teores de Zn pel os extratores $\mathrm{HCl}$ e $\mathrm{M}-1$ e características que representam a capacidade tampão do sol o para a condição de nãoaplicação deZn (Quadro 4) foram significativas, mas não para os extratores DTPA e M-3. Possivel mente, isso se deve ao maior poder de extração do $\mathrm{HCl}$ eM-1, desestabilizando ligações mais estáveis. Com a aplicação de Zn (20 mg dm-3), notou-se inversãonas tendências dessas correlações. Nessa condição, os extratores $\mathrm{HCl}$ e M-1 apresentaram correlações negativas e menores com medidas que estimam a capacidade tampão do solo (Quadro 4), enquanto DTPA e M-3 apresentaram correlações negativas mais el evadas com essas características. Essa inversão deveu-se aparentemente ao maior poder de extração dos extratores $\mathrm{HCl}$ e $\mathrm{M}-1$ e, conseqüentemente, ao menor desgaste desses extratores em condições mais tamponadas, comojá observado para fósforo (Bahia Filho, 1982). Considerando, no entanto, as 
Quadro 3. Zinco extraído por HCl, Mehlich-1 (M-1), Mehlich-3 (M-3) e DTPA das amostras dos solos nas doses de 0 e $20 \mathrm{mg} \mathrm{dm}^{-3}$ de $\mathrm{Zn}$ e as respectivas relações (declividades) entre o $\mathrm{Zn}$ recuperado pelo extrator por unidade de $\mathrm{Zn}$ aplicada $\left(\mathrm{mg} \mathrm{dm}^{-3} / \mathrm{mg} \mathrm{dm}^{-3}\right)$ do modelo linear

\begin{tabular}{|c|c|c|c|c|c|c|c|c|c|}
\hline \multirow{3}{*}{$\begin{array}{c}\text { Solo } \\
\text { CV-AQd }\end{array}$} & \multirow{2}{*}{\multicolumn{2}{|c|}{$\begin{array}{l}\text { Dose } \\
-\mathrm{mg} \mathrm{dm}^{-3}-\end{array}$}} & \multirow{2}{*}{$\frac{\text { Cl }}{\text { Decliv. }}$} & \multicolumn{2}{|c|}{ M-1 } & \multicolumn{2}{|c|}{ M-3 } & \multicolumn{2}{|c|}{ DTPA } \\
\hline & & & & $\mathrm{mg} \mathrm{dm}^{-3}$ & Decliv. & $\mathrm{mg} \mathrm{dm}^{-3}$ & Decliv. & $\mathrm{mg} \mathrm{dm}^{-3}$ & Decliv. \\
\hline & $\begin{array}{r}0 \\
20\end{array}$ & $\begin{array}{r}0,05 \\
16,14\end{array}$ & 0,80 & $\begin{array}{r}0,19 \\
15,83\end{array}$ & 0,78 & $\begin{array}{r}0,45 \\
15,10\end{array}$ & 0,73 & $\begin{array}{l}0,11 \\
8,43\end{array}$ & 0,42 \\
\hline CV-LEm & $\begin{array}{r}0 \\
20\end{array}$ & $\begin{array}{r}0,05 \\
14,78\end{array}$ & 0,74 & $\begin{array}{r}0,23 \\
14,96\end{array}$ & 0,74 & $\begin{array}{r}0,28 \\
11,96\end{array}$ & 0,58 & $\begin{array}{l}0,31 \\
8,02\end{array}$ & 0,39 \\
\hline TM-LEm & $\begin{array}{r}0 \\
20\end{array}$ & $\begin{array}{r}0,00 \\
15,51\end{array}$ & 0,78 & $\begin{array}{r}0,07 \\
15,43\end{array}$ & 0,77 & $\begin{array}{r}0,04 \\
14,26\end{array}$ & 0,71 & $\begin{array}{l}0,06 \\
8,42\end{array}$ & 0,42 \\
\hline T2-LVm & $\begin{array}{r}0 \\
20\end{array}$ & $\begin{array}{r}0,00 \\
16,69\end{array}$ & 0,83 & $\begin{array}{r}0,14 \\
16,74\end{array}$ & 0,83 & $\begin{array}{r}0,09 \\
15,98\end{array}$ & 0,79 & $\begin{array}{l}0,08 \\
8,87\end{array}$ & 0,44 \\
\hline ARA-LA & $\begin{array}{r}0 \\
20\end{array}$ & $\begin{array}{r}0,14 \\
20,04\end{array}$ & 1,00 & $\begin{array}{r}0,19 \\
17,54\end{array}$ & 0,87 & $\begin{array}{r}0,34 \\
18,19\end{array}$ & 0,89 & $\begin{array}{r}0,20 \\
10,05\end{array}$ & 0,49 \\
\hline AÇA-LA & $\begin{array}{r}0 \\
20\end{array}$ & $\begin{array}{r}0,22 \\
19,05\end{array}$ & 0,94 & $\begin{array}{r}0,41 \\
17,95\end{array}$ & 0,88 & $\begin{array}{r}0,70 \\
17,70\end{array}$ & 0,85 & $\begin{array}{r}0,35 \\
11,39\end{array}$ & 0,55 \\
\hline UBE-LVal & $\begin{array}{r}0 \\
20\end{array}$ & $\begin{array}{r}0,62 \\
16,37\end{array}$ & 0,79 & $\begin{array}{r}0,75 \\
16,44\end{array}$ & 0,78 & $\begin{array}{r}0,72 \\
10,85\end{array}$ & 0,51 & $\begin{array}{l}0,28 \\
7,43\end{array}$ & 0,36 \\
\hline UBE-LVa2 & $\begin{array}{r}0 \\
20\end{array}$ & $\begin{array}{r}0,26 \\
16,60\end{array}$ & 0,82 & $\begin{array}{r}0,30 \\
15,63\end{array}$ & 0,77 & $\begin{array}{r}0,16 \\
11,37\end{array}$ & 0,56 & $\begin{array}{l}0,11 \\
8,38\end{array}$ & 0,41 \\
\hline PAR-LV & $\begin{array}{r}0 \\
20\end{array}$ & $\begin{array}{r}0,14 \\
14,68\end{array}$ & 0,73 & $\begin{array}{r}0,27 \\
14,84\end{array}$ & 0,73 & $\begin{array}{l}0,04 \\
8,42\end{array}$ & 0,42 & $\begin{array}{l}0,12 \\
5,84\end{array}$ & 0,29 \\
\hline SL-LE & $\begin{array}{r}0 \\
20\end{array}$ & $\begin{array}{r}0,36 \\
14,08\end{array}$ & 0,69 & $\begin{array}{r}0,50 \\
12,96\end{array}$ & 0,62 & $\begin{array}{l}0,28 \\
7,28\end{array}$ & 0,35 & $\begin{array}{l}0,23 \\
4,17\end{array}$ & 0,20 \\
\hline PAT-LV & $\begin{array}{r}0 \\
20\end{array}$ & $\begin{array}{r}0,58 \\
15,93\end{array}$ & 0,77 & $\begin{array}{r}0,68 \\
15,31\end{array}$ & 0,73 & $\begin{array}{r}0,88 \\
10,32\end{array}$ & 0,47 & $\begin{array}{l}0,26 \\
7,89\end{array}$ & 0,38 \\
\hline$P N-P V$ & $\begin{array}{r}0 \\
20\end{array}$ & $\begin{array}{r}0,57 \\
17,41\end{array}$ & 0,84 & $\begin{array}{r}0,64 \\
15,68\end{array}$ & 0,75 & $\begin{array}{r}1,18 \\
15,29\end{array}$ & 0,71 & $\begin{array}{l}0,38 \\
9,18\end{array}$ & 0,44 \\
\hline M édia & & & 0,81 & & 0,77 & & 0,63 & & 0,40 \\
\hline I.C. $(5 \%)^{(1)}$ & & & $0,78-0,84$ & & $0,74-0,81$ & & $0,57-0,69$ & & $0,37-0,43$ \\
\hline C.V. (\%)(1) & & & 10,63 & & 11,10 & & 26,91 & & 22,56 \\
\hline
\end{tabular}

(1) Intervalo de confiança e coeficiente de variação calculados, utilizando três repetições para todos os solos.

correlações entre as características rel acionadas com a capacidade tampão do solo e as declividades Zn recuperado/Zn adicionado, verificaram-se, de modo geral, maiores valores de correlação para todos os extratores. Comparando entre si os extratores mais sensíveis, DTPA e M-3, percebeu-se maior sensibilidade para o M-3 do que para o DTPA, evidenciada pelo mai or coeficiente de variação para o M-3 (Quadro 3) e pelos maiores coeficientes de correlação entre características dos solos e este extrator (Quadro 4).

Os extratores $\mathrm{HCl}$ e $\mathrm{M}-1$ são os métodos mais amplamente utilizados na determinação do $\mathrm{Zn}$ "disponível", apesar das baixas correlações com o Zn absorvido pelas plantas (Tucker \& Kurtz, 1955; Muraoka et al., 1983; Davis et al., 1995).
Quanto ao fracionamento de $\mathrm{Zn}$ do solo, nos tratamentos sem sua adição ao solo, as frações com as mai ores concentrações em rel ação ao val or total de Zn foram ZnMn eZnFec, para os sol os arenosos, e $\mathrm{ZnF}$ ea e $\mathrm{ZnFec}$, para os solos de textura argilosa (Quadro 5). Para os solos argilosos, o maior teor de goetita (Quadro 2) parece ter sido o principal responsável pela retenção de $Z n$. A relação entre oxidróxidos e passagem de $\mathrm{Zn}$ da forma lábil para não-lábil tem sido amplamente demonstrada pela literatura (E Igabaly, 1950; Hodgson, 1963; Cavallaro \& McBride,1984; Cunha, 1989). Nos sol os arenosos, a quantidade de goetita éconsideravel mentemenor, o que pode explicar a al ocação deZn na fração ZnMn. Fica evidente para o sol o sem a aplicação de Zn que as principais formas de retenção do el emento 


\section{Quadro 4. Correlação de Pearson entre características do solo, fração de zinco residual (Znres) e teores de zinco extraídos por $\mathrm{HCl}$, Mehlich-1 (M-1), Mehlich-3 (M-3) e DTPA e as respectivas declividades ${ }^{(1)}$ para as amostras dos solos e doses de zinco testadas}

\begin{tabular}{|c|c|c|c|c|c|c|c|c|c|c|c|c|c|c|}
\hline Característica ${ }^{(2)}$ & $\begin{array}{l}\text { Dose ou } \\
\text { Decl. Zn }\end{array}$ & Znres. ${ }^{(3)}$ & $\mathrm{HCl}$ & M-1 & M-3 & DTPA & $\mathrm{HCl}$ & M-1 & M-3 & DTPA & $\mathrm{HCl}$ & M-1 & M-3 & DTPA \\
\hline & & & \multicolumn{8}{|c|}{ _ Dose $\left(\mathrm{mg} \mathrm{dm}^{-3}\right)$} & \multicolumn{4}{|c|}{ Declividade $\left(\mathrm{mg} \mathrm{dm}^{-3} / \mathrm{mg} \mathrm{dm}^{-3}\right)$} \\
\hline & & & & & 0 & 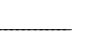 & & -2 & 20 & - & & & & \\
\hline P-remanescente & & $-0,68 * *$ & $-0,51^{*}$ & $-0,48$ & 0,06 & 0,01 & $0,53^{*}$ & $0,58^{*}$ & $0,88 * *$ & $0,74 * *$ & $0,58^{*}$ & $0,64 *$ & $0,88 * *$ & $0,76 * *$ \\
\hline $\mathrm{H}+\mathrm{Al}$ & & $0,71^{* *}$ & 0,48 & 0,48 & $-0,04$ & 0,05 & $-0,59 *$ & $-0,74 * *$ & $-0,90 * *$ & $-0,83^{*} *$ & $-0,64 *$ & $-0,80 * *$ & $-0,91 * *$ & $-0,86 * *$ \\
\hline CTC total & & $0,62 *$ & 0,42 & 0,44 & $-0,05$ & 0,12 & $-0,54 *$ & $-0,71^{* *}$ & $-0,84 * *$ & $-0,79 * *$ & $-0,58 *$ & $-0,76^{* *}$ & $-0,85^{* *}$ & $-0,81^{* *}$ \\
\hline C.O. & & $0,76 * *$ & $0,63^{*}$ & $0,62^{*}$ & 0,12 & 0,09 & $-0,48$ & $-0,49$ & $-0,84 * *$ & $-0,61^{*}$ & $-0,55 *$ & $-0,59 *$ & $-0,87 * *$ & $-0,64 * *$ \\
\hline Argila & & $0,55^{*}$ & $0,54^{*}$ & $0,51^{*}$ & $-0,04$ & 0,05 & $-0,35$ & $-0,46$ & $-0,80 * *$ & $-0,68^{* *}$ & $-0,40$ & $-0,53^{*}$ & $-0,80 * *$ & $-0,70 * *$ \\
\hline C.C. & & $0,67 * *$ & $0,56^{*}$ & $0,54 *$ & 0,00 & 0,02 & $-0,43$ & $-0,50$ & $-0,84 * *$ & $-0,66^{* *}$ & $-0,49$ & $-0,57 *$ & $-0,85^{* *}$ & $-0,68^{* *}$ \\
\hline \multirow[t]{2}{*}{$\mathrm{Gt}^{2 /}$} & & $0,73 * *$ & $0,75^{* *}$ & $0,75^{* *}$ & 0,41 & 0,34 & $-0,55^{*}$ & $-0,64 *$ & $-0,81^{* *}$ & $-0,68^{*}$ & $-0,62^{*}$ & $-0,75^{* *}$ & $-0,85 * *$ & $-0,71^{* *}$ \\
\hline & $\mathrm{mg} \mathrm{dm}^{-3}$ & & & & & & & & & & & & & \\
\hline $\mathrm{HCl}$ & 0 & 0,29 & & $0,98 * *$ & $0,78^{* *}$ & $0,63^{*}$ & 0,03 & $-0,12$ & $-0,34$ & $-0,17$ & $-0,10$ & $-0,30$ & $-0,42$ & $-0,22$ \\
\hline M-1 & 0 & 0,33 & & & $0,80 * *$ & $0,70 * *$ & $-0,02$ & $-0,12$ & $-0,36$ & $-0,18$ & $-0,15$ & $-0,31$ & $-0,44$ & 0,23 \\
\hline$M-3$ & 0 & $-0,08$ & & & & $0,80 * *$ & 0,34 & 0,21 & 0,17 & 0,30 & 0,23 & 0,05 & 0,08 & 0,26 \\
\hline DTPA & 0 & $-0,17$ & & & & & 0,24 & 0,10 & 0,06 & 0,21 & 0,16 & $-0,02$ & $-0,02$ & 0,15 \\
\hline $\mathrm{HCl}$ & 20 & $-0,87 *$ & & & & & & $0,88^{* *}$ & $0,83^{* *}$ & $0,85^{* *}$ & $0,83^{* *}$ & $0,99 * *$ & $0,85^{* *}$ & $0,84 * *$ \\
\hline$M-1$ & 20 & $-0,74 * *$ & & & & & & & $0,84 * *$ & $0,90 * *$ & $0,90 * *$ & $0,89 * *$ & $0,98 * *$ & $0,83^{* *}$ \\
\hline$M-3$ & 20 & $-0,88^{*}$ & & & & & & & & $0,91^{* *}$ & $0,92 * *$ & $0,86 * *$ & $0,87 * *$ & $1,00 * *$ \\
\hline \multirow[t]{2}{*}{ DTPA } & 20 & $-0,79 * *$ & & & & & & & & & $1,00^{* *}$ & $0,86^{* *}$ & $0,90 * *$ & $0,89 * *$ \\
\hline & $\mathrm{dm}^{-3} / \mathrm{mg} \mathrm{dn}$ & & & & & & & & & & & & & \\
\hline $\mathrm{HCl}$ & Decl. & $-0,91 *$ & & & & & & & & & & $0,88 * *$ & $0,85 * *$ & $0,85^{* *}$ \\
\hline$M-1$ & Decl. & $-0,78^{* *}$ & & & & & & & & & & & $0,87 * *$ & $0,91^{* *}$ \\
\hline$M-3$ & Decl. & $-0,89 *$ & & & & & & & & & & & & $0,91^{* *}$ \\
\hline DTPA & Decl. & $-0,78^{* *}$ & & & & & & & & & & & & \\
\hline
\end{tabular}

(1) Declividade ou relação entre o Zn extraído $\left(\mathrm{mg} \mathrm{dm}^{-3}\right)$ em função do Zn aplicado $\left(\mathrm{mg} \mathrm{dm}^{-3}\right)$. (2) C.O. - carbono orgânico; C.C. capacidade de campo, Gt - goetita na fração argila. ${ }^{(3)}$ Znres $=20$ - Znto (somatório de todas as frações de Zn citadas menos a fração residual) na dose $20 \mathrm{mg} \mathrm{dm}^{-3}+$ Znto na dose 0 de $\mathrm{Zn}$ ).

$*$, ** significativos a 5 e a $1 \%$, respectivamente.

encontram-se em frações com ligações mais estáveis, sendo as frações Zntr e Znmo pouco representativas em relação ao total encontrado. Isto também explicaria o comportamento diferencial dos extratores quanto à extração de $\mathrm{Zn}$ do solo na dose zero e de sua correlação com características relacionadas com a capacidade tampão do solo (Quadro 4).

Quando Zn foi aplicado aos solos, a fração que deteve a mai or quantidade desse el emento passou a ser Zntr, observando-se tendência à maior homogeneidade de distribuição de $Z n$ entre as demais frações, principalmente $\mathrm{ZnMn}, \mathrm{ZnF}$ ea e ZnFec (Quadro 5). É importante ressaltar que o tempo necessário para se atingir o equilíbrio de reações do Zn com o solo, após sua aplicação, foi de 30 dias, provavelmente insuficiente para o equilíbrio pleno de todas as reações entre as frações avaliadas.

$\mathrm{O}$ ajuste de regressão múltipla dos teores de Zn pelos extratores, como variáveis dependentes das frações de zinco do solo (Quadro 6), indicou que as frações Zntr, ZnFea e ZnFec contribuíram, de maneira mais efetiva, para o teor de $Z n$ pelos extratores, no solo sem a aplicação de Zn. Nessa situação, as frações de Zn atuaram como fonte de Zn para a extração com $\mathrm{HCl}, \mathrm{M}-1$ e $\mathrm{M}-3$. O mesmo não foi observado em resposta à aplicação de $Z n$, quando aquel as frações, com exceção da Zntr, apresentaramse como dreno desse nutriente. Esse comportamento tão diferente entre os extratores, quando comparados os solos em suas condições naturais e depois de receberem $20 \mathrm{mg} \mathrm{dm}^{-3}$ de $\mathrm{Zn}$, como também verificado pela correlação não-significativa entre os extratores nessa comparação de sem e com a adição deZn (Quadro 4), éindicativo de possível causa para as dificuldades encontradas para se obter uma estimativa confiável de Zn "disponível" do solo pel os métodos utilizados, como já comentado neste trabalho.

Para cada extrator, o grau de importância rel ativa da fração é diferenciado (Quadro 7). No sol os com e sem a aplicação de Zn, a fração trocável éa de maior importância para todos os extratores, respondendo, no mínimo, com a metade do val or de R2 encontrado para todos os ajustes de equação. Semel hantemente, Lindsay (1972) afirmou que o extrator DTPA apresenta íntima correlação com o pool-lábil de Zn no solo ou fator quantidade. 
Quadro 5. Frações de zinco: Zn trocável (Zntr), ligado à matéria orgânica (Znmo), ligado a óxido de manganês (ZnMn), ligado a óxido de ferro amorfo (ZnFea), ligado a óxido de ferro cristalino (ZnFec), residual (Znres.) $)^{(1)}$, total $\left(\right.$ Znto $^{(2)}$ e percentagem relativa ao total encontrado para cada fração nas amostras de solo para as duas doses de zinco

\begin{tabular}{|c|c|c|c|c|c|c|c|c|c|c|c|c|c|c|}
\hline \multirow{2}{*}{ Solo } & Dose de Zn & \multicolumn{2}{|c|}{ Zntr } & \multicolumn{2}{|c|}{ Znmo } & \multicolumn{2}{|c|}{ ZnMn } & \multicolumn{2}{|c|}{ ZnFea } & \multicolumn{2}{|c|}{ ZnFec } & \multicolumn{2}{|c|}{ Znres } & \multirow{2}{*}{$\frac{\text { Znto }}{\mathrm{mg} \mathrm{dm}^{-3}}$} \\
\hline & $-\mathrm{mgdm}^{-3}$ & $i^{-3}-$ & $\%$ & $\mathrm{mg} \mathrm{dm}^{-3}$ & $\%$ & $\mathrm{mg} \mathrm{dm}^{-3}$ & $\%$ & $\mathrm{mg} \mathrm{dm}^{-3}$ & $\%$ & $\mathrm{mg} \mathrm{dm}^{-3}$ & $\%$ & $\mathrm{mg} \mathrm{dm}^{-3}$ & $\%$ & \\
\hline \multirow{2}{*}{ CV-AQd } & 0 & 0,10 & 7,07 & 0,04 & 3,00 & 0,52 & 37,25 & 0,00 & 0,00 & 0,73 & 52,69 & & & 1,38 \\
\hline & 20 & 10,14 & 47,43 & 0,13 & 0,61 & 2,72 & 12,72 & 0,78 & 3,65 & 0,51 & 2,39 & 5,72 & 26,75 & 14,28 \\
\hline \multirow{2}{*}{ CV-LEm } & 0 & 0,10 & 5,87 & 0,00 & 0,00 & 0,23 & 12,84 & 0,00 & 0,00 & 1,41 & 81,28 & & & 1,75 \\
\hline & 20 & 10,53 & 48,41 & 0,21 & 0,97 & 2,38 & 10,94 & 1,22 & 5,61 & 1,23 & 5,66 & 4,42 & 20,32 & 15,58 \\
\hline \multirow{2}{*}{ TM-LEm } & 0 & 0,08 & 7,87 & 0,04 & 4,71 & 0,28 & 30,69 & 0,00 & 0,00 & 0,53 & 56,73 & & & 0,93 \\
\hline & 20 & 10,55 & 50,41 & 0,10 & 0,48 & 2,63 & 12,57 & 0,59 & 2,82 & 0,53 & 2,53 & 5,60 & 26,76 & 14,40 \\
\hline \multirow{2}{*}{ TM-LVm } & 0 & 0,10 & 14,94 & 0,03 & 3,88 & 0,24 & 33,25 & 0,00 & 0,00 & 0,34 & 47,93 & & & 0,71 \\
\hline & 20 & 12,01 & 57,99 & 0,00 & 0,00 & 2,46 & 11,88 & 0,60 & 2,90 & 0,46 & 2,22 & 4,47 & 21,58 & 15,53 \\
\hline \multirow{2}{*}{ ARA-LA } & 0 & 0,27 & 17,26 & 0,07 & 4,09 & 0,47 & 30,04 & 0,04 & 2,19 & 0,71 & 46,41 & & & 1,54 \\
\hline & 20 & 17,40 & 80,78 & 0,00 & 0,00 & 1,60 & 7,43 & 0,61 & 2,83 & 0,39 & 1,81 & 0,01 & 0,05 & 19,99 \\
\hline \multirow{2}{*}{ AÇA-LA } & 0 & 0,44 & 27,30 & 0,07 & 4,10 & 0,44 & 26,83 & 0,00 & 0,00 & 0,68 & 41,78 & & & 1,63 \\
\hline & 20 & 14,97 & 69,21 & 0,06 & 0,28 & 1,55 & 7,17 & 0,46 & 2,13 & 0,68 & 3,14 & 2,28 & 10,54 & 17,72 \\
\hline \multirow{2}{*}{ UBE1-LVa } & 0 & 0,32 & 19,35 & 0,10 & 6,09 & 0,01 & 0,78 & 0,86 & 52,41 & 0,35 & 21,37 & & & 1,64 \\
\hline & 20 & 8,81 & 40,71 & 0,67 & 3,10 & 2,01 & 9,29 & 1,86 & 8,60 & 0,55 & 2,54 & 6,09 & 28,14 & 13,91 \\
\hline \multirow{2}{*}{ UBE1-LVa } & 0 & 0,24 & 29,36 & 0,12 & 14,58 & 0,00 & 0,00 & 0,43 & 53,05 & 0,03 & 3,01 & & & 0,82 \\
\hline & 20 & 9,35 & 44,91 & 0,65 & 3,12 & 1,93 & 9,27 & 1,69 & 8,12 & 0,42 & 2,02 & 5,96 & 28,63 & 14,04 \\
\hline \multirow{2}{*}{ PAR-LV } & 0 & 0,10 & 6,92 & 0,06 & 5,61 & 0,03 & 1,37 & 1,10 & 69,04 & 0,22 & 17,06 & & & 1,50 \\
\hline & 20 & 7,20 & 33,49 & 1,08 & 5,02 & 2,01 & 9,35 & 1,79 & 8,33 & 0,50 & 2,33 & 7,43 & 34,56 & 12,57 \\
\hline \multirow{2}{*}{ SL-LE } & 0 & 0,14 & 6,57 & 0,24 & 10,42 & 0,03 & 1,27 & 1,07 & 47,83 & 0,75 & 33,92 & & & 2,23 \\
\hline & 20 & 6,54 & 29,42 & 2,87 & 12,91 & 1,73 & 7,78 & 1,88 & 8,46 & 0,83 & 3,73 & 6,15 & 27,67 & 13,85 \\
\hline \multirow{2}{*}{ PAT-LV } & 0 & 0,29 & 18,58 & 0,02 & 1,18 & 0,04 & 2,05 & 1,00 & 63,96 & 0,23 & 14,23 & & & 1,58 \\
\hline & 20 & 8,16 & 37,81 & 0,67 & 3,10 & 1,80 & 8,34 & 1,92 & 8,90 & 0,44 & 2,04 & 7,01 & 32,48 & 12,99 \\
\hline \multirow{2}{*}{ PN - PV } & 0 & 0,46 & 19,03 & 0,09 & 3,51 & 0,51 & 21,23 & 0,30 & 12,40 & 1,05 & 43,83 & & & 2,40 \\
\hline & 20 & 12,38 & 55,27 & 0,51 & 2,28 & 2,53 & 11,29 & 0,96 & 4,29 & 0,73 & 3,26 & 2,89 & 12,90 & 17,11 \\
\hline
\end{tabular}

(1) Znres = 20 - (Znto na dose $20 \mathrm{mg} \mathrm{dm}^{-3}+$ Znto na dose $\left.0 \mathrm{mg} \mathrm{dm}^{-3}\right)_{\text {.(2) }}^{(2)}$ Somatório de todas as frações.

A importância relativa das demais frações variou de acordo com o poder de extração do extrator. Para $\mathrm{M}-1$, na dose de $20 \mathrm{mg} \mathrm{dm}^{-3}$, o coeficiente de regressão da fração Zntr apresentou a menor significância $(11,7 \%)$. I sso provavel mente se deve à covariância dessa variável com ZnMn; as duas variáveis apresentaram um coeficiente de correl ação de 0,78, muito próximo aovalor anteriormentecitado como ponto de referência para a eliminação de uma das variáveis do modelo.
Outra variável que poderia explicar a presença deZn nos extratores seria a Znres. Sua importância pode ser observada pela sua el evada correlação com extratores e algumas características do solo (Quadro 4). Essa variável, no entanto, também apresentou elevada covariância com Zntr, o que impediu sua utilização para o ajuste de regressão. De acordo com os valores cal culados para a falta de ajustamento do modelo (Quadro 7), as equações, excetuando para o $\mathrm{HCl}$ e o $\mathrm{M}-1$ nos sol os sem a 
Quadro 6. Equações de regressão múltipla do teor de zi inco pelos extratores, $\mathrm{HCl}$, Mehlich-1 (M-1), Mehlich-3 (M-3) e DTPA em função das frações de zinco no solo: trocável (Zntr), ligado à matéria orgânica (Znmo), ligado a óxido de manganês (ZnMn), ligado a óxido de ferro amorfo (ZnFea) e ligado a óxido de ferro cristalino (ZnFec)

\begin{tabular}{|c|c|c|c|}
\hline Extrator & Dose Zn & E quação & $\mathbf{R}^{2}$ \\
\hline & $\mathrm{mg} \mathrm{dm} \mathrm{m}^{-3}$ & & \\
\hline $\mathrm{HCl}$ & 0 & $\hat{\mathrm{Y}}=-0,0940+1,2355^{* *} \mathrm{Zntr}+0,2198^{* *} \mathrm{ZnF}$ ea & 0,73 \\
\hline$M-1$ & 0 & $\hat{Y}=-0,0080+1,1710^{* *}$ Zntr $+0,2070^{* * Z n F}$ ea $+0,1196 * * Z n F$ ec & 0,70 \\
\hline$M-3$ & 0 & $\hat{\mathrm{Y}}=-0,280^{* *}+2,4891^{* *} \mathrm{Zntr}+0,3201^{* * Z \mathrm{ZnFec}}$ & 0,80 \\
\hline DTPA & 0 & $\hat{Y}=0,0170+0,6284^{* * Z n t r}-0,2870^{* *} Z n m o-0,1426^{* *} Z n M n+0,1693^{* * Z n F ~ e c}$ & 0,76 \\
\hline $\mathrm{HCl}$ & 20 & $\hat{\mathrm{Y}}=14,50^{* *}+0,4536^{* *} \mathrm{Zntr}-1,8694^{* *} \mathrm{ZnF}$ ec $-0,8378^{* *} \mathrm{ZnMn}$ & 0,87 \\
\hline$M-1$ & 20 & $\hat{Y}=18,99^{* *}+0,1008^{(1)} \mathrm{Zntr}-1,1161^{* * Z n m o}-1,2349 *$ ZnMn $-1,4830 * * \mathrm{ZnFec}$ & 0,68 \\
\hline$M-3$ & 20 & $\hat{\mathrm{Y}}=12,02^{* *}+0,5054^{* *} \mathrm{Zntr}-0,7444^{* *} \mathrm{Znmo}-2,6020^{* *} \mathrm{ZnF}$ ea $-1,3332^{* *} \mathrm{ZnFec}$ & 0,96 \\
\hline DTPA & 20 & $\hat{\mathrm{Y}}=7,57^{* *}+0,2587^{* *} \mathrm{Zntr}-1,3961^{* *} \mathrm{Znmo}-0,6383^{* *} \mathrm{ZnMn}$ & 0,88 \\
\hline
\end{tabular}

(1) Significativos a 11,7\%; *,** significativos a 5 e a 1\%, respectivamente.

Quadro 7. Valores de $\mathbf{R}^{2}$ parciais para os coeficientes significativos dos modelos de regressão múltipla, para os extratores $\mathrm{HCl}$, Mehlich-1 (M-1), Mehlich-3 (M-3) e DTPA, nas doses de zinco testadas, e teste F para a falta de ajustamento dos modelos

\begin{tabular}{|c|c|c|c|c|c|c|c|c|}
\hline Extrator & Dose Zn & $\mathbf{R}^{2}$ total & Zntr & Znmo & ZnMn & ZnFea & ZnFec & F - falta ajustamento \\
\hline \multicolumn{3}{|c|}{$\mathrm{mg} \mathrm{dm}^{-3}$} & \multicolumn{5}{|c|}{$-R^{2}$ parcial } & \\
\hline $\mathrm{HCl}$ & 0 & 0,73 & 0,532 & - & - & 0,201 & - & $4,06 * *$ \\
\hline$M-1$ & 0 & 0,70 & 0,539 & - & - & 0,130 & 0,032 & $2,96 *$ \\
\hline$M-3$ & 0 & 0,80 & 0,729 & - & - & - & 0,083 & $0,09^{\text {ns }}$ \\
\hline DTPA & 0 & 0,76 & 0,511 & - & 0,033 & - & 0,194 & $0,91^{\text {ns }}$ \\
\hline $\mathrm{HCl}$ & 20 & 0,87 & 0,761 & - & 0,037 & - & 0,075 & $0,53^{\text {ns }}$ \\
\hline$M-1$ & 20 & 0,68 & 0,434 & 0,058 & 0,070 & & 0,116 & $0,89^{\text {ns }}$ \\
\hline$M-3$ & 20 & 0,96 & 0,836 & 0,019 & - & 0,094 & 0,008 & $0,76^{\text {ns }}$ \\
\hline DTPA & 20 & 0,88 & 0,723 & 0,144 & 0,014 & - & - & $3,71^{* *}$ \\
\hline
\end{tabular}

ns, $*, * *$ não-significativo, significativos a 5 e a $1 \%$, respectivamente.

aplicação de zinco, e DTPA na dose de $20 \mathrm{mg} \mathrm{dm}^{-3}$, não necessitaram de outro ajuste matemático além do apresentado, uma vez quea falta de ajustamento dos model os não foi significativa.

\section{CONCLUSÕES}

1. A fração deZn trocável foi a maior responsável pela quantidade de $Z n$ obtida pelos extratores. As frações de Zn trocável, Zn ligado à matéria orgânica, a óxidos de manganês, a óxidos de ferro amorfo e a ferro cristalino não foram suficientes para explicar, em todos os casos, o Zn recuperado pelos extratores.

2. Os extratores DTPA e M ehlich-3 foram os mais sensíveis a características dos sol os relacionadas com a capacidade tampão (fator capacidade de Zn no solo). 


\section{LITERATURA CITADA}

AHUMADA, T.I .; BUSTAMANTE, A. \& SCHALSCHA, E.B. Zinc speciation in phosphate-affected soils. Comm. Soil Sci. Plant Anal., 28:989-995, 1997.

AKRAM, M.; CHAUDHRY, R.A.; AHMAD, Z. \& HAQ, G.U. Prediction DTPA soil test zinc and associated rice response to applied zinc. Comm. Soil Sci. Plant Anal., 26:259-268, 1995.

ALVAREZ V., V.H. Caracterização química de solos. Viçosa, Universidade Federal de Viçosa, 1985. 77p.

ALVAREZ V., V.H. Efecto de los factores cantidad, intensidad y capacidad amortiguadora de fosfatos em la evaluación del fósforo disponible de suel os derivados de cenizas vol cánicas de la Meseta Tarasca. Chapingo: Col egio de Posgraduados, 1982. 300p. (Tese de Doutorado)

BAHIA FILHO, A.F.C. Índices da disponibilidade de fósforo em Latossol os do planalto central com diferentes características texturais e mineralógicas. Viçosa, U niversidade F ederal de Viçosa, 1982. 179p. (Tese de Doutorado)

BAR-YOSEF, B. pH-dependent zinc adsorption by soils. Soil Sci. Soc. Am. J ., 43:1095-1099, 1979.

BRAY, R.P. \& KURTZ, L.T. Determination of total organic and available forms of phosphorus in soil. Soil Sci., 59:39-45, 1945.

CAVALLARO, N. \& McBRIDE, M.B. Zinc and copper sorption and fixation by an acid soil clay: Effect of selective dissolutions. Soil Sci. Soc. Am. J ., 48:1050-1055, 1984.

CHOWDHURY, A.K.; MCLAREN, R.G.; CAMERON, K.C. \& SWIFT, R.S. Fractionation of zinc in some New Zealand Soils. Comm. Soil Sci. Plant Anal., 28:301-312, 1997.

COUTO, C.; NOVAIS, R.F.; TEIXEIRA, J .L.; BARROS, N.F. \& NEVES, J .C.L. Níveis críticos de zinco no solo e na planta para o crescimento de milho em amostras de solos com diferentes valores de fator capacidade. R. Bras. Ci. Solo, 16:79-87, 1992.

CUNHA, R.C.A. Retenção e movimento de zinco em solos do estado de São Paulo. Piracicaba, Escola Superior de Agricultura Luiz deQueiroz, 1989. 115p. (Tese de Mestrado)

DAVIS, J .G.; GAINES, T.P. \& PARKER, M.B. Comparison of soil zinc extractants for detection of applied zinc and prediction of leaf zinc concentration. Comm. Soil Sci. Plant Anal., 26:2969-2981, 1995.

DEFELIPO, B.V. \& RIBEIRO, A.C. Análise química de solo: Metodologia. Viçosa, U niversidade F ederal de Viçosa, 1981. 17p. (Boletim de Extensão, 29)

ELGABALY, M.M. Mechanism of zincadsorption by colloidal clays and related minerals. Soil Sci., 69:167-73, 1950.

EMPRESA BRASILEIRA DE PESQUISA AGROPECUÁRIA EMBRAPA. Serviço Nacional de Levantamento e Conservação de Solos. Manual de métodos de análise de solos. Rio deJ aneiro, 1979. não paginado.

FERNANDES, B. \& SYKES, J .O. Capacidade de campo eretenção de água em três solos de Minas Gerais. R. Ceres, 15:1-39, 1968.
FERNÁNDEZ R., I.E.J . Reversibilidade de fósforo não-lábil em diferentes solos, em condições naturais e quando submetidos à redução microbiológica ou química. Viçosa, Universidade Federal de Viçosa 1994. 93p. (Tese de Doutorado)

HODGSON, J .F. Chemistry of themicronutrient elements in soils. Adv. Agron., 15:119-159, 1963.

KALBASI, M.; RACZ, G.J . \& LOEWEN-RUDGERS, L.A. Mechanism of zinc adsorption by iron and aluminium oxides. Soil Sci., 125:146-150, 1978.

BRASIL - Ministério da Agricultura. Análise de corretivos fertilizantes e inoculantes. Métodos oficiais. Brasília: LANARV/SNAD, 1983. 103p.

LINDSAY, W.L. \& NORVELL, W.A. Developmente of a DTPA test for zinc, iron, manganese, and copper. Soil Sci. Soc. Am. J ., 42:421-428, 1978.

LINDSAY, W.L. Inorganic phase equilibria of micronutrients in soils. In: MORTVEDT, J . ., ed. Micronutrients in agriculture. Madison, Soil Science Society of America, 1972. p.41-57.

MEHLICH, A. Mehlich-3 soil test extractant: A modification of Mehlich-2 extractant. Comm. Soil Sci. Plant Anal., 15:1409$1416,1984$.

MENEZES, A.A. Disponibilidade de zinco, para milho, pelos extratores Mehlich-1, Mehlich-3 e DTPA. Viçosa, Universidade Federal de Viçosa, 1997. 147p. (Tese de Mestrado)

MURAOKA, R.; NEPTUNE, A.M.L. \& NASCIMENTO FILHO, V.F. Avaliação da disponibilidade de zinco e manganês do solo para o feijoeiro. I. Zinco. R. Bras. Ci. Solo, 7:167-175, 1983.

NOVAIS, R.F. \& SMYTH, T.J. Fósforo em solo e planta em condições tropicais. Viçosa, U niversidade F ederal de Viçosa, 1999. 399p.

RESENDE, M.; BAHIA FILHO, A.F.C. \& BRAGA, J.M. Mineralogia de Latossolos estimada por alocação a partir doteor total deóxidos do ataque sulfúrico. R. Bras. Ci. Solo, 11:1-23, 1987

SIMS, J .T. Soil pH effects on the distribution and plant availability of manganese, copper and zinc. Soil Sci. Soc. Am. J ., 50:367373,1986

SINGH, R.S.; SING, R.P.; RAI, R.K. \& AGRAWAL, H.P. Relationship between soil test and uptake of copper and zinc by grasses on polluted soil. Comm. Soil Sci. Plant Anal., 25:1313-1320, 1994.

SOON, Y.K. Changes in forms of soil zinc after 23 years of cropping following clearing of a boreal forest. Can. J . Soil Sci., 74:179-184, 1994.

TUCKER, T.C. \& KURTZ, L.T. A comparison of several methods with the bio-assay procedure for extraction of zinc from soils. Soil Sci. Soc. Proc., 19:477-481, 1955.

VETTORI, L. Métodos de análise de solo. Rio deJ aneiro, E.P.E., 1969. 24p. (Boletim Técnico, 7)

WU, X.; AASEN , I \& SELMER-OLSEN, A.R. A study of extraction methods for assessing soil zinc availability: I. Soil zinc extractability and soil zinc buffering capacity in relation to soil properties. Nor. J . Agric. Sci., 5:89-107, 1991. 